\title{
Characterization of a Laser Scanner Sensor for the Use as a Reference System in Vehicular Relative Positioning
}

\author{
Fabian de Ponte Müller ${ }^{1}$, Luis Martín Navajas², and Thomas Strang ${ }^{1}$ \\ 1 Institute of Communications and Navigation, German Aerospace Center, Munich, \\ Germany \\ 2 University of Málaga, Málaga, Spain
}

\begin{abstract}
Advanced Driver Assistance Systems (ADAS) play an important role in increasing the safety on today's roads. Forward collision warning systems, lane change assistants or cooperative adaptive cruise control are examples of safety relevant applications that rely on accurate relative positioning between vehicles. Current solutions found in commercial automobiles estimate the position of surrounding vehicles by measuring the distance with RADAR, cameras or IR-sensors. It is envisioned that the advent of inter-car communication will provide onboard relative positioning systems with further information about other vehicles in the surrounding area. While performing research in this field, the need of a proper reference system for testing new approaches originates. In the ideal case, such a reference system would yield the exact and continuous 3D baseline between two vehicles at any time in any circumstance. In this paper we will characterize the use of a laser scanner as a reference system for relative vehicle positioning.
\end{abstract}

Keywords: Laser Scanner, LD-MRS, Relative Positioning, RTK, Vehicles

\section{Introduction}

The knowledge about other vehicle's position is a fundamental prerequisite for numerous Advanced Driver Assistance Systems (ADAS) in the Intelligent Transportation Systems (ITS) domain. Specially many safety relevant applications require robust relative positioning of surrounding vehicles rather than an absolute position of vehicles on the globe. Forward Collision Warning (FCW) or Cooperative Adaptive Cruise Control (CACC) are examples of such ITS safety applications. Relative positioning in traffic environment is typically addressed using a ranging sensor as for instance a RADAR. Laser scanners, due to their high cost, are currently not considered as a mass market solution in the automotive industry. Camera systems, on the other hand, are a promising solution that is still under research. However, single sensor solutions might not always meet the requirements imposed by future safety applications. For this reason numerous research groups currently work on sensor fusion approaches where the information 
from different sources is combined. The future availability of a wireless communication link between vehicles enables to extend the vehicle's perception range and develop cooperative approaches to estimate the target vehicle's position by combining sensors from different vehicles.

When evaluating the designed relative positioning algorithms in a real world environment, developers use a variety of systems to determine the "true" range between the vehicles. The system under test is then compared with the reference using this value. However, the employed reference systems are often not suited for the stated purpose. In some cases the chosen system lacks the required accuracy or a position accuracy analysis is not properly undertaken. In other cases the reference system cannot be universally employed, as it is only locally available and thus reduces the evaluation to a certain scenario. Also situations have been recorded where due to an unappropriate selection of the reference system test runs with an insufficient statistic were evaluated.

This paper presents a collection of different reference systems found in the literature stating their advantages and disadvantages by analyzing various parameters like accuracy, range, availability, price, etc. A solution for a relative positioning reference system by employing a laser scanner is presented in detail and compared to the previous systems. The laser scanner reveals certain important advantages over the mentioned systems and these have been verified experimentally in a set of tests.

The paper is structured as follows: The following section gives an overview of possible reference systems for vehicle relative positioning by analyzing approaches published by different research groups in this field. The third section presents the laser scanner in detail as a further reference system for vehicle relative positioning analyzing its advantages and disadvantages over the aforementioned systems. The laser scanner has been characterized for its accuracy, reliability and scanning range in a set of measurements. The experimental setup and its results are presented in section four. The paper ends with a conclusion.

\section{Current Approaches}

There are several systems that can be used as a reference system to evaluate new positioning approaches. When evaluating absolute or relative localization systems the key parameter characterizing the reference system is the accuracy in the position. A reference system might also give a measurement for the speed vector of the tracked object, absolute or relative. Further performance parameters are the coverage range, the sight line, the dynamic performance, the measuring rate, synchronization ability and the latency.

Although this paper will discuss a reference systems for relative localization of vehicles, we will first list different approaches for measuring the ground truth in a global or local coordinate frame. By duplicating any of the following systems and differencing its absolute positions the relative baseline between two vehicles can be retrieved. However, the relative position or speed accuracy, will fall off in quality by "adding" the position or speed error. 
Global Navigation Satellite Systems (GNSS), like the US Global Positioning System (GPS) or the European Galileo system, can be used as a rough reference system when evaluating rough positioning techniques. In multipath free environments and with good sky visibility GNSS position accuracy usually lies below $10 m$ [1]. Satellite based augmentation systems like the European EGNOS system can improve this accuracy by having geostationary satellites broadcasting correction signals. Many research groups favor to use Differential GPS (DGPS) with a local base station broadcasting pseudorange corrections. The position accuracy in the horizontal plane is in the order of $1 \mathrm{~m}$ [1]. For instance [2] uses a DGPS approach to evaluate a radio ranging technique based on the received signal strength. In [3] differential GPS is used as a ground truth when comparing two movement models for vehicles and for determining the true lateral location of lanes by performing repeated runs. [4] compares a vehicle trajectory estimation algorithm to WAAS-GPS, the US equivalent to EGNOS.

Real Time Kinematic (RTK) systems can offer a far better solution for the absolute position than using stand-alone or differential GPS. In Real Time Kinematic (RTK) systems the carrier phase to the satellites in view is tracked and, along with the measurements of a reference base station (real or virtual, online or post-processed), the carrier phase ambiguity is solved. In case of a correct carrier resolution towards at least four satellites, this method is able to yield position accuracies in the order of $1 \%$ of the GPS wavelength, i.e. $2 \mathrm{~mm}$ [1].

Schubert et. al. present in [5] a series of movement models which were evaluated with experimental data. A DGPS receiver with RTK capability was used for calculating the reference trajectory of the vehicle. The performance of an RTK receiver highly depends on its capability of resolving the unknown integer number of carrier cycles from the satellite to the receiver. Only in case of a fix ambiguity resolution the stated sub-centimeter accuracy can be achieved. Schubert's group took this into account during evaluation and discarded test runs with non-fix solutions. However, in multipath environments it is possible that the RTK device might be tracking the phase of a reflected signal and therefore give a wrong position solution. Also Alam et. al. [6] use this technology to acquire a reference for the relative position of two vehicles. During their measurement run they encountered the carrier-fix issue and could finally only utilize a 12 minute period out of a 45 minute journey for evaluation. To assess an Ultra-wideband system, in [7] GPS and GLONASS carrier phase measurements are post-processed along with IMU data using Waypoint's Inertial Explorer and GrafNav software packages. The resulting absolute positions of two vehicles are used to compute the bearing and range between them whenever a fixed ambiguity solution is found. In [8] and [9] two further examples for carrier phase based relative positioning by differencing absolute positions can be found.

GNSS based systems have the advantage of being globally available and offering a position solution in global coordinates. When moving from stand-alone GNSS, to SBAS, to DGPS and to RTK the accuracy of the system increases, so does the cost of the system. The time to fix increases while the lock robustness degrades. DGPS and RTK might need a reference station and a permanent 
communication link to the target vehicle. The systems have a good performance under clear-sky conditions but suffer severely in obstacle rich scenarios and therefore are unsuited for urban measurements.

Infrastructure based techniques might also offer advantages in determining the absolute position of a vehicle. Huang et al. [10] used an automated vehicle with on-board magnetometers following a track of magnetic markers. The paper states a lateral accuracy of $3 \mathrm{~cm}$ without mentioning the accuracy, if even available, along the magnetic track. The clear disadvantage of techniques based on fix infrastructure is their limited deployability to different scenarios.

A further solution for tracking the absolute position of a vehicle is to use an optical measurement equipment. Tachymeters, commonly used in surveying, calculate the polar coordinates of a target prism by comparing phase shift measures from a reflected laser beam. A servomotor control system makes it possible to automatically track the target. If the geographic position of the tachymeter is known, the absolute position of the target prism can be computed. The usage of the tachymeter as a reference system is limited by its range and requires constant line of sight between the device and target vehicle. This limits its application to wide obstacle-free areas. As a kinematic measuring device it is limited in speed to around $20 \mathrm{~m} / \mathrm{s}$ and in angular velocity to around $45^{\circ} / \mathrm{s}$. Its high update rate of over $10 \mathrm{~Hz}$ and its sub-centimeter accuracy are clear advantages of this system. Schönber at. al. use this technique to evaluate their GPS/INS solution for an autonomous vehicle in [11].

When it comes to determine the relative baseline between two land vehicles one alternative would be to duplicate any of the above listed absolute position sensors. However, the errors of both systems will add in quadrature (assuming independent errors). One simple solution is to force a static baseline and compare the system under test against the constant known range. In [12] Travis et al. use a towed trailer to force a constant distance between two GNSS antennas for evaluating a differential RTK approach for estimating the relative position between vehicles. The immediate drawbacks of this system are the limits in baseline length and driving speed. To avoid the problem of error growth, a solution that measures directly the baseline between the vehicles is preferred. A laser scanner sensor, the chosen device to be used as a reference system by our research group, will be analyzed in the following sections.

\section{Laser Scanner}

Our experiments aim to proof the usage of the SICK LD-MRS laser scanner as a reference sensor for relative positioning. For this purpose the maximum range and the range accuracy of the scanner are addressed. In the scope of this text the range vector is defined in a coordinate system centered at the foremost center point in the detector vehicle with the $\mathrm{x}$-axis pointing in the driving direction and the y-axis to the left of the vehicle (see Fig. 1). The laser scanner's measurement frame is co-located and aligned with this frame (see Fig. 2). The range vector points to the rearmost center point of the target vehicle. 


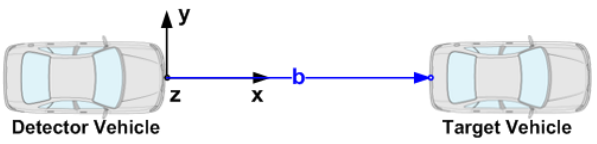

Fig. 1.

The LD-MRS is a four layer laser scanner which uses a rotating mirror and several pulsed laser beams to calculate the distance towards reflecting objects based on time of flight (TOF) technology. The angular resolution of the laser scanner is dependent on the scanning frequency, which is configurable to $12.5 \mathrm{~Hz}$, $25 \mathrm{~Hz}$ and $50 \mathrm{~Hz}$. At the default $12.5 \mathrm{~Hz}$ frequency, an angular resolution of $0.5^{\circ}$ is achieved on each layer. The scanning aperture ranges from $50^{\circ}$ to $-60^{\circ}$.

The product sheet states a maximum range of $50 \mathrm{~m}$ with $10 \%$ remission and $160 \mathrm{~m}$ for $100 \%$ remission surfaces (the light reflecting from a perfectly diffuse reflecting white surface corresponds to the definition of 100\%). The data sheet further states a measurement resolution of $40 \mathrm{~mm}$ and range error $(1 \sigma)$ of $100 \mathrm{~mm}$. These values are going to be verified in the following experiments.

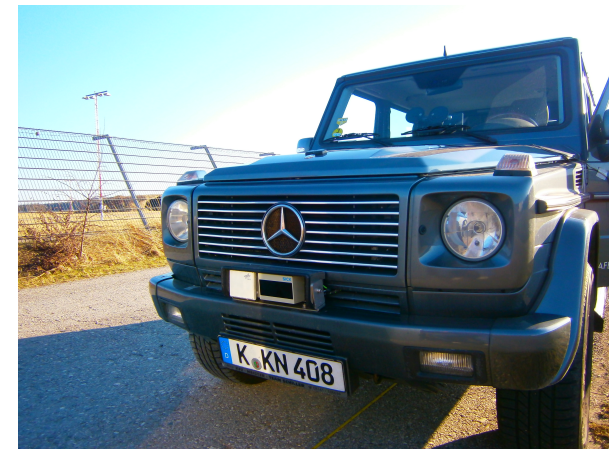

Fig. 2. SICK LD-MRS laser scanner mounted on the SOL-Car.

The laser scanner outputs both scan data as well as object data. The scan data is the raw measurement including the distance of each measurement point on each layer along with the echo pulse width in $\mathrm{cm}$. One abstraction layer above, the object data contains the result of the detection and tracking of single objects out of the clouds of scan points. Objects are either localized by a reference point, their contour line or their bounding box. Fig. 4 shows an example of a static scan on a parking lot. The corresponding environment is shown in Fig. 3.

By using the object data from the sensor for relative positioning of vehicles the work of detecting and tracking objects is already done in the sensor, thus shortening the development time and decreasing the processing requirements on the application unit. In Fig. 4 it can be observed how clouds of scan points 


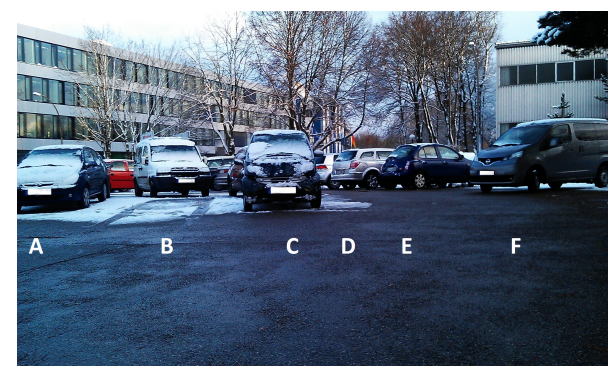

Fig. 3. View from the laser scanner.
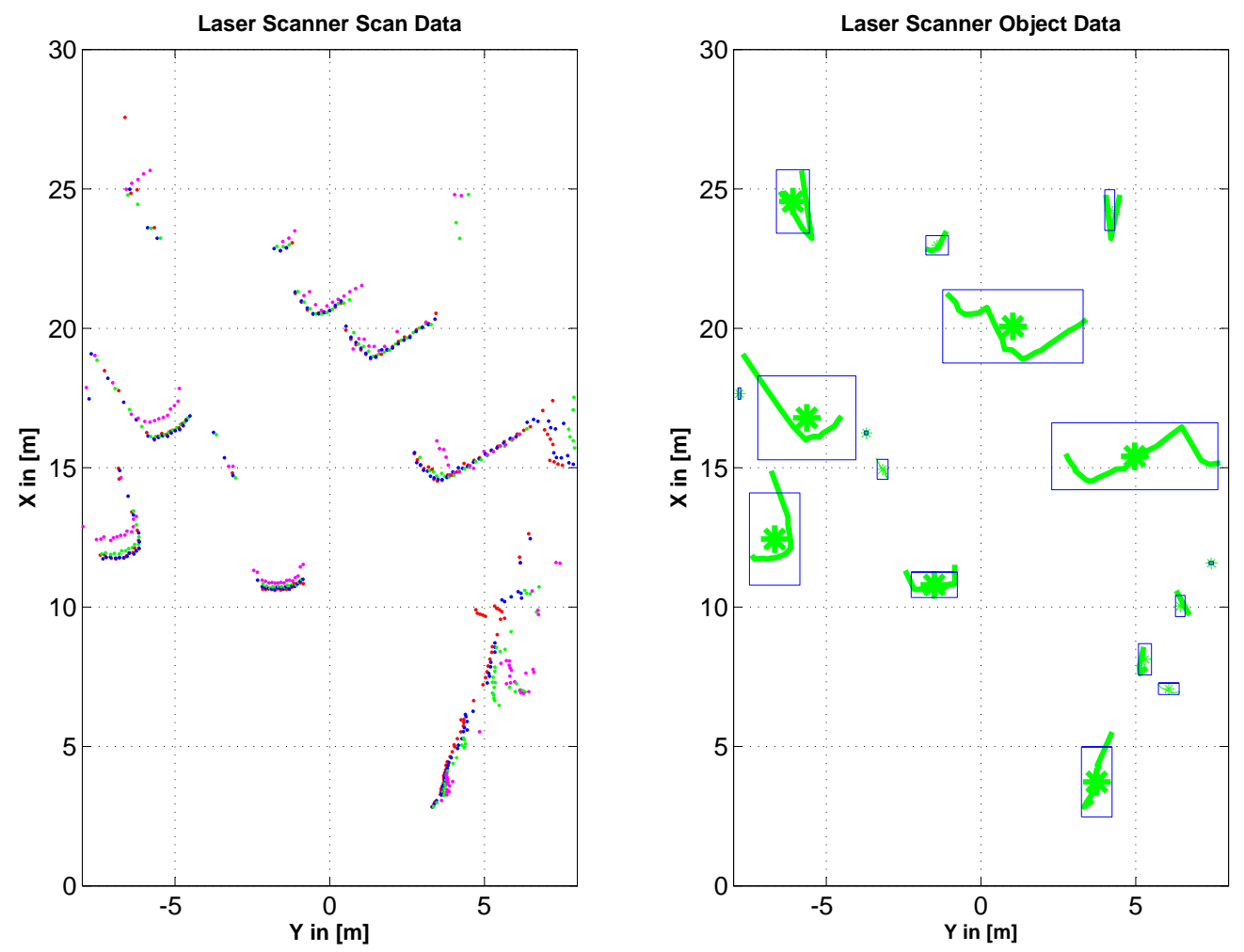

Fig. 4. Output of the SICK LD-MRS scanner: raw scan data and object data 
are clustered into single detected objects. The front line of the reflection points generate the contour line (green line) and its center of mass is taken as the reference point for the object position (green asterisk). However, the usage of this data comes along with certain drawbacks that should be taken into account.

It can be noticed that nearby target objects might not be correctly resolved and merged into the same detected object (e.g. vehicles D and E). However, this behavior was rarely noticed in real driving environments. Fig. 4 also reveals a circumstance occurring when a target vehicle is in an oblique position to the sensor (e.g. vehicle F). Such geometry between two vehicles can be observed in real traffic in bends, roundabouts or intersections. The displacement of the center of mass of the contour line will introduce a systematic error in the object's position estimate, which will depend on the dimensions and the relative position of the target vehicle and its relative heading towards the detector vehicle.

Also in scenarios with an in-front placed target object a series of considerations have to be made. Depending on the shape of the rear end of the vehicle the measurement point might be displaced longitudinally by up to several decimeters. At short distances several scanning planes will hit the rear side of the vehicle. The resulting contour line will more likely coincide with its actual distance (e.g. vehicle B). However, when increasing the distance, only one of the scanning planes might hit the target vehicle and thus producing an unknown offset (see Fig. 5).

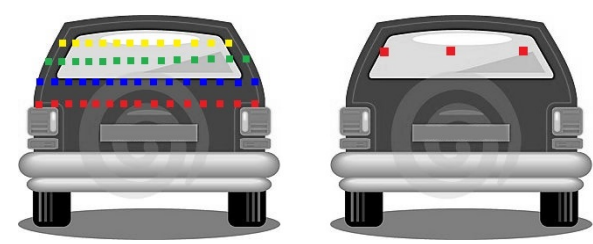

Fig. 5. Sketch showing the scanning planes hitting on the rear side of a vehicle. The left shows a nearby vehicle hit by four planes. the right picture shows a distant vehicle hit by only one plane.

Further on, with increasing distance the scanning points get laterally further separated leading to fewer points measuring the position of the target vehicle. Whilst at $20 \mathrm{~m}$ distance and measuring with two planes the points are separated $8 \mathrm{~cm}$ from each other, at $100 \mathrm{~m}$ the points are as far as $87 \mathrm{~cm}$ by measuring with only one plane. The number of laser points actually measuring the distance has a direct impact on the lateral position resolution.

Table 1 lists the theoretical expected error in lateral direction due to the limited angular resolution. The number of scanning planes and points per distance have been retrieved from a test run with a $1800 \mathrm{~mm}$ wide and $1500 \mathrm{~mm}$ high target vehicle. These values, along with the longitudinal position accuracy stated in the data sheet $(0.1 \mathrm{~m})$ can be taken as the best performance that can be expected from the sensor. 


\begin{tabular}{|l|l|l|l|l|l|l|l|}
\hline Distance & $10 \mathrm{~m}$ & $20 \mathrm{~m}$ & $30 \mathrm{~m}$ & $50 \mathrm{~m}$ & $80 \mathrm{~m}$ & $100 \mathrm{~m}$ & $120 \mathrm{~m}$ \\
\hline Number of Planes & 4 & 4 & 3 & 1 & 1 & 1 & 1 \\
\hline Hor. Angular Resolution & $0.25^{\circ}$ & $0.25^{\circ}$ & $0.25^{\circ}$ & $0.5^{\circ}$ & $0.5^{\circ}$ & $0.5^{\circ}$ & $0.5^{\circ}$ \\
\hline Hor. Point Distance & $0.04 \mathrm{~m}$ & $0.08 \mathrm{~m}$ & $0.13 \mathrm{~m}$ & $0.43 \mathrm{~m}$ & $0.69 \mathrm{~m}$ & $0.87 \mathrm{~m}$ & $1.04 \mathrm{~m}$ \\
\hline Number of Points on Target & 40 & 20 & 13 & 4 & 3 & 2 & 1 \\
\hline Maximum lateral Error at $0^{\circ}$ & $0.02 \mathrm{~m}$ & $0.03 \mathrm{~m}$ & $0.05 \mathrm{~m}$ & $0.19 \mathrm{~m}$ & $0.2 \mathrm{~m}$ & $0.4 \mathrm{~m}$ & $0.37 \mathrm{~m}$ \\
\hline Maximum lateral Error at $30^{\circ}$ & $0.03 \mathrm{~m}$ & $0.08 \mathrm{~m}$ & $0.15 \mathrm{~m}$ & $0.27 \mathrm{~m}$ & $0.40 \mathrm{~m}$ & $0.31 \mathrm{~m}$ & $0.50 \mathrm{~m}$ \\
\hline
\end{tabular}

Table 1. Theoretical maximum lateral error in dependence of the distance.

The next section presents a series of measurement runs performed to determine the accuracy of the laser scanner sensor in terms of its bias and standard deviation.

\section{Evaluation}

A series of measurement runs have been performed to understand and characterize the behavior of the sensor in real world situations. The static measurements were performed with a vehicle equipped with a SICK LD-MRS four-layer laser scanner under test mounted on its front bump. The laser scanner is connected via Ethernet to the on-board automotive computer, which receives the detected objects at a rate of $12.5 \mathrm{~Hz}$. For each object its position in the form of $\mathrm{x} / \mathrm{y}$ coordinates in the vehicle's body frame along with the estimated line representing its contour are output.

A reflector has been used as the target object. Its rectangular shape, with $1400 \mathrm{~mm}$ width and $1500 \mathrm{~mm}$ height, and the material, aluminum, resemble that of a vehicle. The plain surface of the reflector enables to measure the distance unambiguously, as the laser beam reflects directly on its surface, unlike a vehicle where the reflection point cannot be determined definitely.

The environment in which the measurement run was performed consisted of a straight single lane road inside the premises of DLR. The left side of the road is free of obstacles, while on the right side a metallic fence runs along the road. The fence, as well as other obstacles located behind the target vehicle that fell into the detection range of the laser scanner, produce objects that had to be filtered out manually. Several measurement procedures have been used to assess the laser scanner performance in such scenario.

Tape Measure A tape measure was used to determine the distance between the reflector and the vehicle. The tape was laid perpendicularly to the reflector keeping the center of the laser scanner always on top of it. As the maximum length of the tape was $50 \mathrm{~m}, 22$ measuring point were taken between 0 and $40 \mathrm{~m}$. At each measuring point the vehicle was stopped between 5 to 15 seconds, which corresponds to 60 to 180 laser scanner object detections. For each point the distance between reflector and vehicle has been measured "by hand" using the tape measure. From the large list of detected objects the one corresponding 
to the reflector is taken, its distance is calculated using the law of cosines and compared to the distance measured by hand.

For each point measured with the tape measure the laser error bias and its standard deviation are calculated. Fig. 6 shows these values for the 22 measured points. The values lie between 1 and $6 \mathrm{~cm}$, what is as well inside the range of the stated laser scanner's resolution and in the order of the accuracy of the measurement technique.

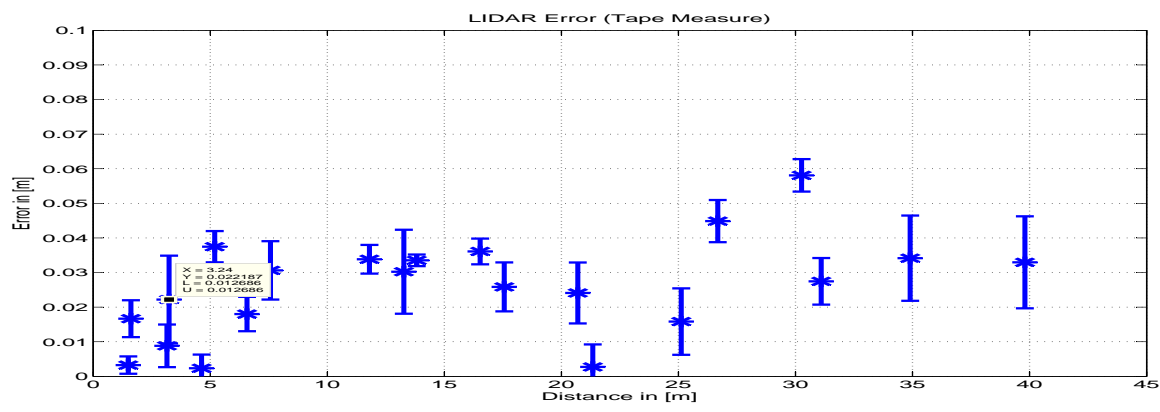

Fig. 6. Bias and standard deviation for different distances compared to a tape measure.

GNSS Carrier phase Measurement A second test series using RTK carrier phase solution was chosen. On both the reflector and on the front of the detector vehicle a GNSS antenna was placed. The distance between the vehicle and the reflector was changed from 0 to $50 \mathrm{~m}$. At each measuring point the vehicle was stopped for several minutes to ensure that a carrier phase solution would be achieved during post processing. Also the movements between two different measurements were done smoothly and slowly to avoid losing carrier phase fix. For the absolute geo-position of the reflector in earth-centered-earth-fixed (ECEF) coordinates over the measurement period of 53 minutes a standard deviation of $\sigma_{x}=0.0082 m, \sigma_{y}=0.0072 m$ and $\sigma_{z}=0.0120 m$ was measured, suggesting a lock on the carrier phase. The average over the measurement period yields the position of the reflector. The vehicle's position is converted to the ECEF frame, the baseline is calculated by subtracting both coordinates and the norm of the vector yields the distance from the vehicle to the reflector. After subtracting the antenna offset to the laser scanner sensor $(0.3 \mathrm{~m})$, both the distance between the correct detected object and the relative distance calculated by subtracting the RTK positions is compared to each other. 14 measurement points were taken from $1.5 \mathrm{~m}$ to $45 \mathrm{~m}$ distance. Fig. 7 shows again the resulting bias and standard deviation in each measurement. The figure shows laser scanner errors between $0 \mathrm{~cm}$ and $8 \mathrm{~cm}$ when compared to RTK. This error is in the order of the previous measurements performed with the measuring tape, thus validating both approaches and giving an idea of the expected laser scanner error in this range. 


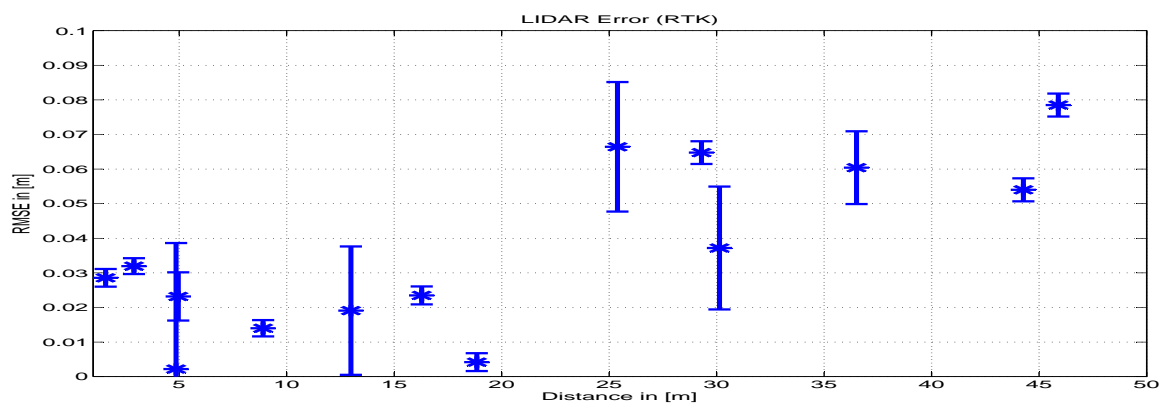

Fig. 7. Bias and standard deviation for different distances compared to the differenced RTK positions.

Tachymeter A TCRP 1201 tachymeter from Leica has been used to measure the position of the target vehicle with respect to the laser scanner. Internally the tachymeter measures the range, azimuth and elevation angles towards a reflector point. To precisely orient the tachymeter a set of points have been taken on the detector vehicle. A prism was located on the roof on the rear part of the target vehicle. In post-processing the retrieved coordinates of the prism were translated and rotated to transpose them from the global coordinate frame to the sensor's coordinate frame of the laser scanner. The offset between the tachymeter and the most rear point of the vehicle $(20 \mathrm{~cm})$ were corrected.

The stated accuracy of the tachymeter ( $2 \mathrm{~mm}$ in range and $0.27 \mathrm{mdeg}$ in angle) is achieved under static conditions, whereas in tracking mode, measurements in range and angle might not be synchronized and the resulting coordinates and their associated timestamps would be inconsistent [13]. For these reasons, only static phases where the target vehicle was standing still were taken for evaluation. Fig. 8 shows the along-track and cross-track components for the laser scanner (blue) and tachymeter (green) in dependence of time. The distance between the vehicles was increased stepwise from $6 \mathrm{~m}$ to about $120 \mathrm{~m}$ and decreased again down to $7 \mathrm{~m}$. The cross-track distance varied in a range of $1.5 \mathrm{~m}$.

The measurement run consisted of 35 points in the range of $0 \mathrm{~m}$ to $120 \mathrm{~m}$. Fig 9 displays the resulting along-track bias and standard deviation for each of the measurements.

A minor offset of less than two centimeters can be observed at short distances. This offset is increased to about $15 \mathrm{~cm}$ and is maintained from $15 \mathrm{~m}$ to $60 \mathrm{~m}$. From this distance on, and coinciding with the measurement from only one laser plane, the offset varies from about $10 \mathrm{~cm}$ to $40 \mathrm{~cm}$ along with an increased standard deviation. The fact that this measurement run was performed using a vehicle as a target object instead of the reflector can explain the increased error in comparison to the previous experiments using the tape measure and and RTK. Further on, it can be observed that the cross-track error is marginal for short distances and increases with distance. At a distance above $100 \mathrm{~m}$ this error is about $1 \mathrm{~m}$, matching the theoretical analysis performed in the previous sections. 

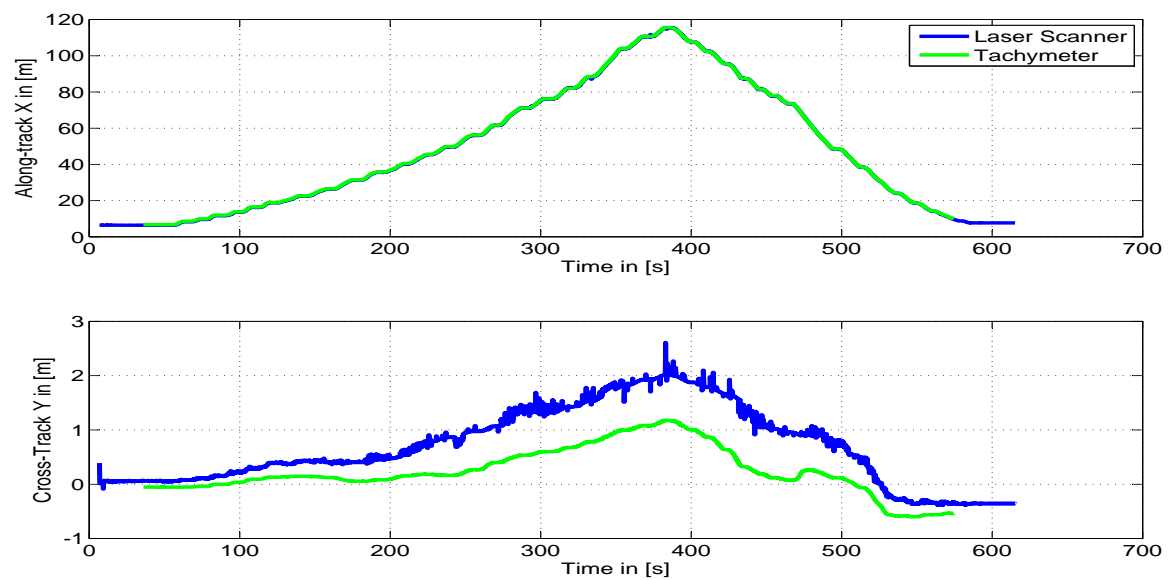

Fig. 8. Along-track and cross-track coordinate for laser scanner (blue) and tachymeter (green).

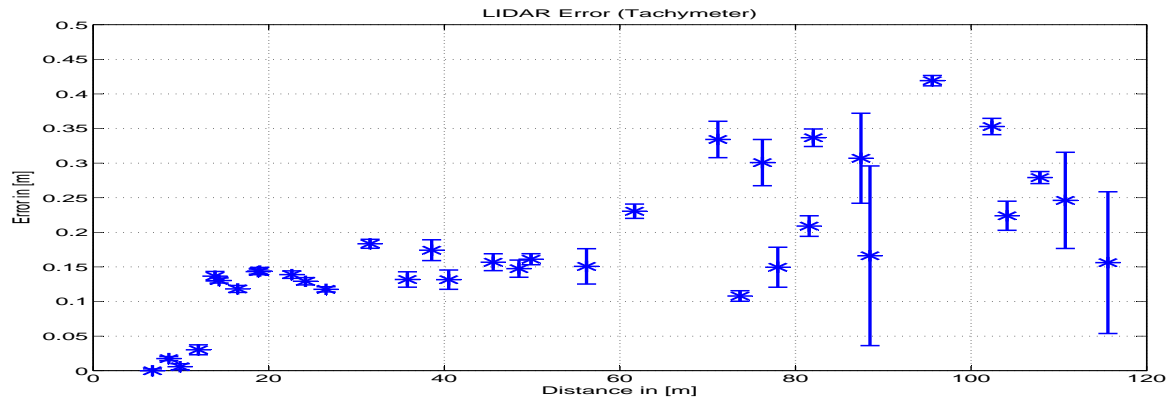

Fig. 9. 35 measuring points comparing the laser scanner along track coordinate to the correspondent point measured with the Leica Tachymeter. 


\section{General Conclusion}

The authors conclude that the laser scanner is a convenient and relatively inexpensive solution to be used as a reference system for vehicle relative positioning. This paper has listed the particularities of the sensor, its drawbacks and special characteristics that have to be taken into account for its usage in static scenarios. The ranging accuracy of the laser scanner sensor has been characterized in static scenarios by using different techniques including a tape measure, a GNSS carrier phase based positioning engine and a tachymeter. It has been shown that the distance accuracy of the laser scanner is below $10 \mathrm{~cm}$ in the range of 0 to $50 \mathrm{~m}$. The shape of the rear of the target vehicle introduces an unknown range ambiguity due to the variation in the measuring point. Further on, it has been shown that the limited angular resolution of the laser scanner leads to a lateral position error when increasing the distance.

To conclude we present the following recommendations when the object data from the laser scanner is to be used as a reference system for relative vehicle position:

- With distance the longitudinal accuracy of the laser scanner degrades. Up to $60 \mathrm{~m}$ an accuracy of about $15 \mathrm{~cm}$ could be verified.

- With distance the lateral accuracy degrades due to the limited angular resolution.

- In bends the object's center point is displaced from the vehicle's true center point. Therefore, we recommend using the device for tracking the in-front driving vehicle in straight maneuvers.

- Using a vehicle with a planar rear end is recommended to avoid errors caused to measurements at different heights.

- The use of wider vehicles is also recommended, in order to guarantee a certain lateral accuracy at higher distances.

\section{Acknowledgements}

The authors would like to thank EDMO-Flugbetrieb GmbH for the opportunity to run the experiments under almost ideal GNSS coverage conditions and for their safe coordination of accessing the runway alternating with normal air traffic.

\section{References}

1. E. Kaplan, Understanding GPS - Principles and applications. Artech House, 2nd edition ed., December 2005.

2. V. Kukshya, H. Krishnan, and C. Kellum, "Design of a system solution for relative positioning of vehicles using vehicle-to-vehicle radio communications during GPS outages," in Vehicular Technology Conference, 2005. VTC-2005-Fall. 2005 IEEE 62nd, vol. 2, pp. 1313-1317, Jan. 2006. 
3. S. Rezaei and R. Sengupta, "Kalman filter based integration of DGPS and vehicle sensors for localization," in Mechatronics and Automation, 2005 IEEE International Conference, vol. 1, pp. 455 - 460 Vol. 1, july-1 aug. 2005.

4. Z. Kim and M. Cao, "Evaluation of feature-based vehicle trajectory extraction algorithms," in Intelligent Transportation Systems (ITSC), 2010 13th International IEEE Conference on, pp. 99 -104, sept. 2010.

5. R. Schubert, E. Richter, and G. Wanielik, "Comparison and evaluation of advanced motion models for vehicle tracking," in Information Fusion, 2008 11th International Conference on, pp. 1 -6, 30 2008-july 32008.

6. N. Alam, A. Tabatabaei Balaei, and A. G. Dempster, "Relative positioning enhancement in VANETs: A tight integration approach," Intelligent Transportation Systems, IEEE Transactions on, vol. PP, no. 99, pp. 1 -9, 2012.

7. M. Petovello, K. O'Keefe, and B. Chan, "Demonstration of inter-vehicle UWB ranging to augment DGPS for improved relative positioning," in Proceedings of IEEE/ION GNSS 2010, 2010.

8. C. Basnayake, C. Kellum, J. Sinko, and J. Strus, "GPS-based relative positioning test platform for automotive active safety systems," in Proceedings of the 19th International Technical Meeting of the Satellite Division of The Institute of Navigation (ION GNSS 2006), 2006.

9. N. Luo and G. Lachapelle, "Relative positioning of multiple moving platforms using gps," Aerospace and Electronic Systems, IEEE Transactions on, vol. 39, pp. 936 948 , july 2003.

10. J. Huang and H.-S. Tan, "A low-order DGPS-based vehicle positioning system under urban environment," Mechatronics, IEEE/ASME Transactions on, vol. 11, pp. $567-575$, oct. 2006 .

11. T. Schönberg, M. Ojala, J. Suomela, A. Torpo, and A. Halme, "Positioning an autonomous off-road vehicle by using fused dgps and inertial navigation," in In 2nd IFAC Conference on Intelligent Autonomous Vehicles, pp. 226-231, 1995.

12. W. Travis and D. Bevly, "Trajectory duplication using relative position information for automated ground vehicle convoys," in Position, Location and Navigation Symposium, 2008 IEEE/ION, pp. 1022 -1032, May 2008.

13. W. Stempfhuber and T. Wunderlich, "Auf dem Weg zur Sensorsynchronisation von GPS und TPS für kinematische Messaufgaben," Allgemeine VermessungsNachrichten (AVN), vol. 111, pp. 175-184, May 2004. 